\title{
SIMULATION STUDY FOR THE SEPARATION OF RARE ISOTOPES AT THE SEOUL NATIONAL UNIVERSITY AMS FACILITY
}

\author{
C C Yun $•$ C S Lee $1,2 \cdot \mathrm{M} \mathrm{Youn}^{3} \cdot \mathrm{J} \mathrm{C} \mathrm{Kim}^{3}$
}

ABSTRACT. A simulation study for the separation of rare isotopes such as beryllium and aluminum was performed for a new beam line to be attached to the 3MV Tandetron accelerator at the accelerator mass spectrometry (AMS) facility of Seoul National University in Korea. The new beam line will also be used for other scientific applications, namely, ion implantations, Rutherford backscattering, and nuclear astrophysics experiments. It mainly consists of $30^{\circ}$ and $100^{\circ}$ deflection dipole magnets and drift spaces. A transfer matrix for the beam line was determined by the TRANSPORT code. Simulation of the rare isotope separation was performed by a ray tracing method using the TURTLE code. The simulation results, including the effect of the energy degrader, provide feasibility for the separation of isobars with small mass differences in ${ }^{10} \mathrm{Be}-{ }^{10} \mathrm{~B}$ and ${ }^{26} \mathrm{Al}-{ }^{26} \mathrm{Mg}$.

\section{INTRODUCTION}

Accelerator mass spectrometry (AMS) is a powerful technique for measuring long-lived radionuclides that occur naturally in our environment. It has been used for a wide variety of dating and tracing applications in the geological and planetary sciences, archaeology, biomedicine, etc. The AMS facility of Seoul National University (SNU-AMS) in Korea was completed in December 1998 (Kim et al. 2000). It uses a Tandetron accelerator of the Cockroft-Walton type, manufactured by High Voltage Engineering Europa in Holland, and has a dedicated radiocarbon AMS beam line, as well as 5 ports available for other experimental purposes. It uses a cesium sputtering ion source for ionizing carbon isotopes as well as other species. A recombinator system in the injection part of the SNU-AMS allows 3 carbon isotopes, ${ }^{12} \mathrm{C},{ }^{13} \mathrm{C}$, and ${ }^{14} \mathrm{C}$, to be simultaneously measured. A detailed description of the system including detection and analysis procedures is found in Kim et al. (2001). The recent status and future plan of the SNU-AMS was presented at a symposium in October 2002 (Kim et al. 2003).

The main mission of the SNU-AMS thus far has been to count carbon isotopes. Recently, a new project to measure other radionuclides has begun with the design of a new beam line (see Figure 1). As an initial step, we decided to focus on the measurement of beryllium isotopes, ${ }^{9} \mathrm{Be}$ (a stable nuclide) and ${ }^{10} \mathrm{Be}$ (a radionuclide with a half-life of 1.5 million yr), and of aluminum isotopes, ${ }^{27} \mathrm{Al}$ (a stable nuclide) and ${ }^{26} \mathrm{Al}$ (a radionuclide with a half-life of 0.73 million yr). The ${ }^{10} \mathrm{Be}$ and ${ }^{26} \mathrm{Al}$ radionuclides are produced in meteorites or in other extraterrestrial materials, in the atmosphere of the earth, and on the surface of the earth. They can, therefore, serve as valuable tracers, a chronological clock for prehistoric samples. In this paper, we present the simulation results, including the effect of the energy degrader through a new beam line previously reported by Yun et al. (2003).

\section{DESIGN AND SIMULATION FOR THE NEW BEAM LINE}

The new beam line was designed by the TRANSPORT code (Brown et al. 1973), which is based on a matrix approach. Transport properties of a charged particle beam can be simulated by defining a beam vector in this code. The beam vector can be written as

\footnotetext{
${ }^{1}$ Department of Physics, Chung-Ang University, Seoul 156-756, Korea.

${ }^{2}$ Corresponding author. Email: cslee@cau.ac.kr.

${ }^{3}$ Department of Physics, Seoul National University, Seoul 151-742, Korea.
}

(C) 2004 by the Arizona Board of Regents on behalf of the University of Arizona Proceedings of the 18th International Radiocarbon Conference, edited by N Beavan Athfield and R J Sparks RADIOCARBON, Vol 46, Nr 1, 2004, p 89-95 


$$
X(0)=\left(\begin{array}{c}
x \\
x^{\prime} \\
y \\
y^{\prime} \\
\ell \\
\delta_{0}
\end{array}\right)
$$

The beam vector consists of 6 components corresponding to the horizontal $(x)$ and vertical $(y)$ beam extents in units of $\mathrm{cm}$; the horizontal $\left(x^{\prime}\right)$ and vertical $\left(y^{\prime}\right)$ beam divergences or angles in units of mrad; the longitudinal beam extend $(\ell)$ in units of $\mathrm{cm}$; and finally, the momentum spread $\left(\delta_{0}\right)$ in units of $\%$ for the charged particle transported through the beam line.

The transfer matrix $R$ at a specific position can be represented by

$$
X(1)=R X(0)
$$

where $X(0)$ is the beam vector at an initial point. $X(1)$ is the beam vector at a specific position. When there are $n$ components for the beam line, $R$ in Equation 2 can be rewritten as a product of matrices corresponding to each component:

$$
R(t)=R(n) \ldots R(3) R(2) R(1)
$$

where $R(i)(i=1,2, \ldots . n)$ can represent a dipole magnet, a quadrupole element, the drift space, a shim angle, etc.

The new beam line (Figure 1) consists of a $30^{\circ}$ deflection (D1) and a $100^{\circ}$ deflection (D2) dipole magnet previously used as a gas-filled recoil mass separator and as a beam analyzer, respectively, at the SF cyclotron facility at the Center for Nuclear Study, the University of Tokyo. The basic parameters of the 2 dipole magnets are given in Table 1 . The energy degrader is situated on the beam axis inside the scattering chamber located between 2 dipole magnets. A Faraday cup is also situated inside the scattering chamber but off the beam axis. The dispersion $D$ listed in Table 1 represents separability between particles of different momenta at a magnet exit, and can be obtained from the following relation:

$$
D=\frac{\rho(1-\cos \theta)}{100}
$$

where the dispersion $D$ is in units of $\mathrm{cm} / \%$ and $\rho$ and $\theta$ are the central radius and the deflection angle of a dipole magnet, respectively. The resolving power of a beam line system can be written as (Livingood 1969):

$$
R_{p}=\frac{P}{\Delta P}=\frac{D^{\prime}}{2 x_{0} M_{x}}
$$

where $x_{0}$ and $M_{x}$ are the initial beam size and the magnification, respectively. The relationship between the dispersion of the individual magnets and the overall beam line dispersion $D^{\prime}$ is a function of the beam line geometry. Therefore, the maximum resolving power should be carefully determined by taking into account the positions of the 2 dipole magnets and the drift space in the new beam line. 


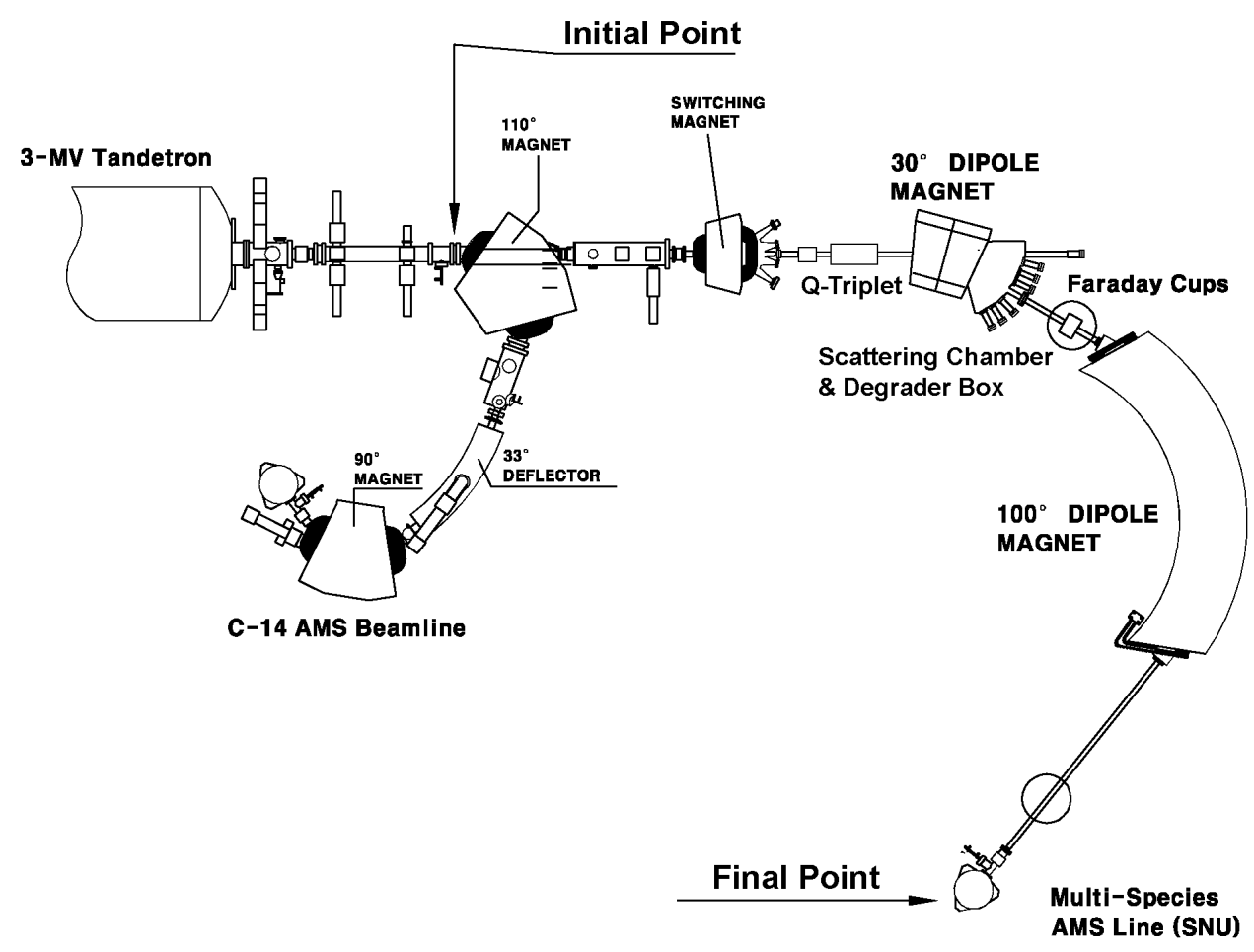

Figure 1 Schematic view of the SNU-AMS facility

Table 1 Beam-optical parameters of the D1 and D2 dipole magnets.

\begin{tabular}{lll}
\hline Parameters & D1 & D2 \\
\hline Deflection angle $\left({ }^{\circ}\right)$ & 30 & 100 \\
Central radius $(\mathrm{cm})$ & 134.2 & 180 \\
Pole gap $(\mathrm{cm})$ & 5.6 & 7.0 \\
Entrance and exit angle $\left(^{\circ}\right)$ & - & 30.8 \\
Maximum field $(\mathrm{T})$ & 1.22 & 0.74 \\
Maximum $B \rho(\mathrm{Tm})$ & 1.6 & 1.33 \\
Dispersion $(\mathrm{cm} / \%)$ & 0.18 & 2.11 \\
\hline
\end{tabular}

On the other hand, the mass resolving power of the beam line system can be obtained from the following relationship:

$$
\mathrm{R}_{\mathrm{M}}=\frac{1}{2} \mathrm{R}_{\mathrm{p}}
$$

For the initial beam parameters in the subsequent calculation, we used the values supplied by the manufacturer of the SNU-AMS for the horizontal $\left(\varepsilon_{x}\right)$ and the vertical $\left(\varepsilon_{y}\right)$ emittance and the momentum spread $\left(\delta_{0}\right)$. They were $\varepsilon_{x}=3.0 \pi \mathrm{mm} \bullet \mathrm{mrad}(0.5 \mathrm{~mm} \times 6.0 \mathrm{mrad}), \varepsilon_{y}=3.0 \pi \mathrm{mm} \cdot \mathrm{mrad}$ $(1.2 \mathrm{~mm} \times 2.5 \mathrm{mrad})$ in $3-\sigma$ emittance, and $\delta_{0}=0.01 \%$, respectively. To simulate the trajectory of a charged particle through the magnetic field, we performed computation using a ray tracing method including higher order aberration, called TURTLE (Trace Unlimited Rays Through Lumped Elements) (Brown et al. 1974). The initial beam vector was created assuming the Gaussian distribution using the random number generator. 
The 3- $\sigma$ emittance $\varepsilon_{x}$ can be obtained by

$$
\varepsilon_{x}=\left[\left(3 \sigma_{x}\right)^{2}\left(3 \sigma_{x^{\prime}}\right)^{2}-\left(3 \sigma_{x} \times 3 \sigma_{x^{\prime}}\right)^{2}\right]^{1 / 2}
$$

where $\sigma_{x}$ and $\sigma_{x}^{\prime}$ are the standard deviations corresponding to the horizontal beam extent $x$ and angle $x^{\prime}$, respectively. The standard deviations are found in the general manner that

$$
\sigma=\sqrt{\frac{1}{N} \sum_{i=1}^{N}\left(x_{i}-\bar{x}\right)^{2}}
$$

where $\bar{x}$ is the average of $x_{i}$ of each point.

The energy loss in degrading material was obtained by the Bethe-Bloch equation for higher order corrections. The energy straggling was determined using the method of Tschalär (Tschalär et al. 1968). The multiple scattering was calculated as follows (Highland 1975):

$$
\sigma(\theta)=\frac{14.1 M e V l c}{\sqrt{p_{i} \beta_{i} p_{f} \beta_{f}}} Z_{i} \sqrt{\left(\Delta x / L_{r}\right)}\left[1+\frac{1}{9} \log \frac{\Delta x}{L_{r}}\right]
$$

where the angular width is given in terms of the radiation length $\left(L_{r}\right)$ and the initial and final momenta $\left(p_{i}\right.$ and $\left.p_{f}\right)$ and velocities $\left(\beta_{i} c\right.$ and $\left.\beta_{f} c\right)$. The values of the radiation length for aluminum and beryllium are $8.9 \mathrm{~cm}$ and $35.28 \mathrm{~cm}$, respectively (Tsai 1974). The parameters used in the present simulation for beryllium-boron isotopes were assumed to have the energy of $10 \mathrm{MeV}$ and the charge state of $3^{+}$. For aluminum-magnesium isotopes, the energy of $12.5 \mathrm{MeV}$ and the charge state of $4^{+}$ were assumed. A total of 5000 particles were considered in all the simulation.

\section{RESULTS AND DISCUSSION}

Figure 2 shows the result obtained by the TRANSPORT code for the region from the initial point to the final point (schematically shown in Figure 1). The top and bottom panels represent the beam envelope and the dispersion, respectively, for the new beam line. The upper half-plane of each panel represents the vertical beam transport while the lower half-plane represents the horizontal beam transport. The rectangles in the figure indicate a triplet quadrupole lens and dipole magnets. As shown in the bottom panel of Figure 2, the dispersion at the point of Faraday cups in Figure 1 turned out to be $0.49 \mathrm{~cm} / \%$, whereas the dispersion at the final point was $7.74 \mathrm{~cm} / \%$. From equations (5) and (6), the resolving powers at this point are $R_{P} \sim 2000$ and $R_{M} \sim 1000$, which sets the limit of separation to be $\sim 0.05 \%$ in the momentum difference and $\sim 0.1 \%$ in the mass difference.

Figure 3 shows the result of separation between beryllium-boron isotopes of the TURTLE code obtained by the transfer matrix from the TRANSPORT code. The 3 figures illustrate, from left to right, the $\mathrm{x}-\mathrm{y}$ beam profile at the initial point, before the energy degrader, and at the final point, respectively. The values for $\varepsilon_{x}$ and $\varepsilon_{y}$ were 3.03 and $2.98 \pi \mathrm{mm} \bullet \mathrm{mrad}$, respectively, at the initial point. The right figure illustrates the beam profile after the energy degrader at the final point. The values of ${ }^{10} \mathrm{~B}$ for $\varepsilon_{x}$ and $\varepsilon_{y}$ were 159.14 and $63.65 \pi \mathrm{mm} \cdot \mathrm{mrad}$, respectively. The values of ${ }^{10} \mathrm{Be}$ for $\varepsilon_{x}$ and $\varepsilon_{y}$ were 104.17 and $50.07 \pi \mathrm{mm} \bullet \mathrm{mrad}$, respectively. The beam spot size of ${ }^{10} \mathrm{~B}$ at final point was $7.82 \mathrm{~cm} \times 5.76 \mathrm{~cm}$ in the $\mathrm{x}-\mathrm{y}$ beam profile. For the ${ }^{10} \mathrm{Be}$ isotope, the beam spot size at the final point was $6.16 \mathrm{~cm} \times 5.11 \mathrm{~cm}$ in the $\mathrm{x}-\mathrm{y}$ beam profile. 

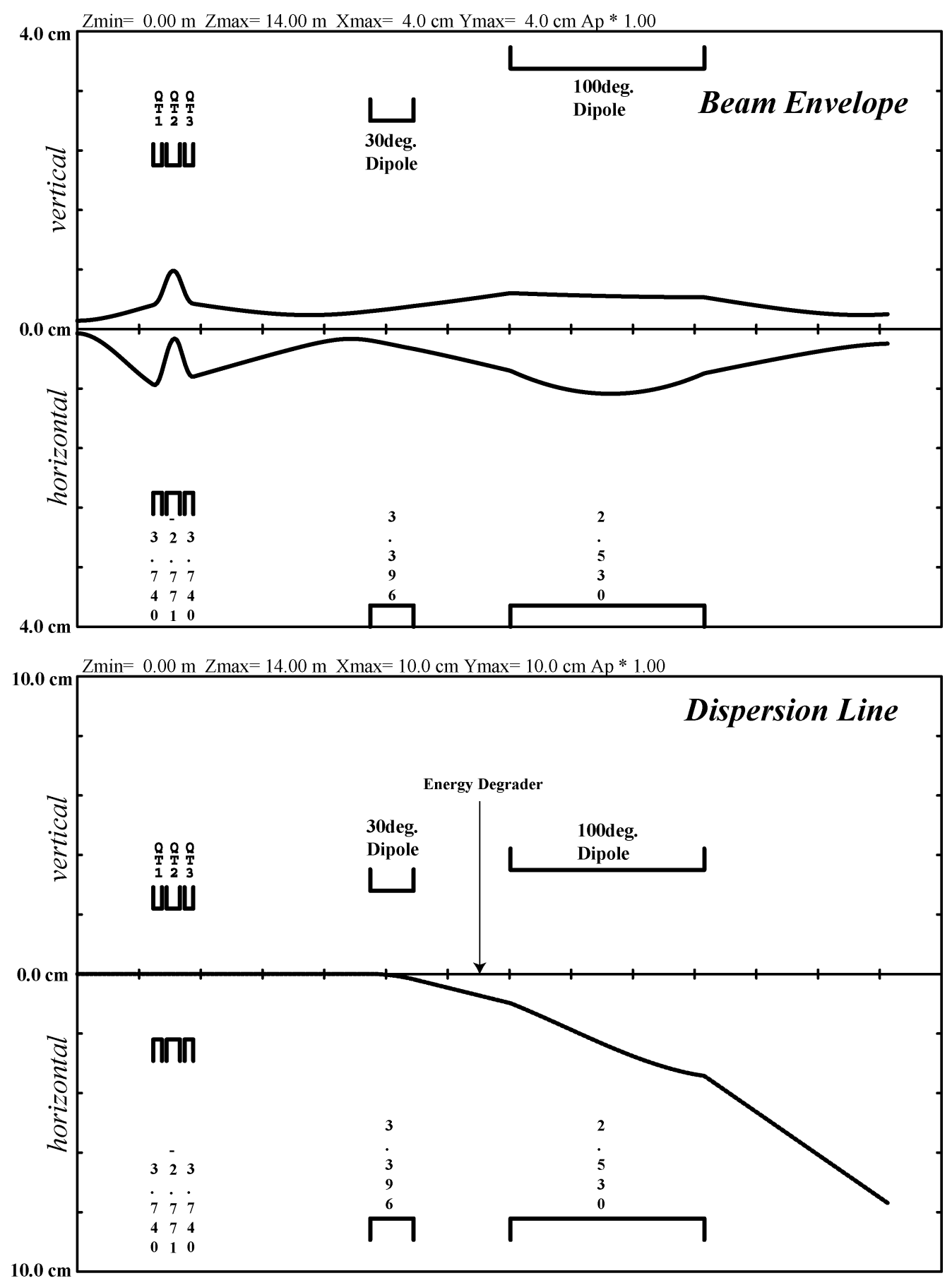

Figure 2 Beam envelope (top panel) and dispersion (bottom panel) calculated in the present work. The calculation results were plotted for the beam transport range from the initial point to the final point shown in Figure 1. 

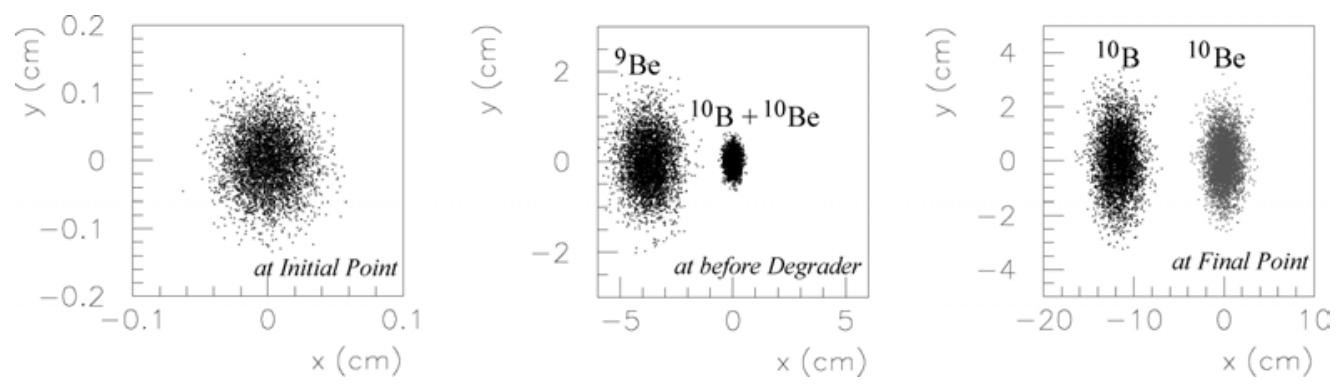

Figure 3 Result for separation of beryllium isotopes obtained by the TURTLE code. Explanations for these plots are given in the text.

As seen from the middle plot in Figure 3, ${ }^{10} \mathrm{Be}$ is clearly separated from ${ }^{9} \mathrm{Be}$. However, a bundle of admixed ${ }^{10} \mathrm{Be}$ and ${ }^{10} \mathrm{~B}$ is inseparable despite the large dispersion value of $7.74 \mathrm{~cm} / \%$ at the final point. This results from the limit of separation as mentioned above: the mass difference between ${ }^{10} \mathrm{~B}$ and ${ }^{10} \mathrm{Be}$ amounts was a mere $0.006 \%$, so the momentum difference is only $0.003 \%$. On the other hand, the isotopes passing through the energy degrader have a different energy for the different charge number $(Z)$ because the energy loss is proportional to $Z^{2}$ of the isotopes. The energies of ${ }^{10} \mathrm{~B}$ and ${ }^{10} \mathrm{Be}$ were $9.253 \mathrm{MeV}$ and $9.557 \mathrm{MeV}$, respectively, after the energy degrader with a $290 \mu \mathrm{g} / \mathrm{cm}^{2}$ $(1 \mu \mathrm{m})$ aluminum foil. This amount of $3.28 \%$ in energy difference corresponds to $1.64 \%$ in the momentum difference. This momentum difference leads to the separation of $12.7 \mathrm{~cm}$ between ${ }^{10} \mathrm{~B}$ and ${ }^{10} \mathrm{Be}$ at the final position.
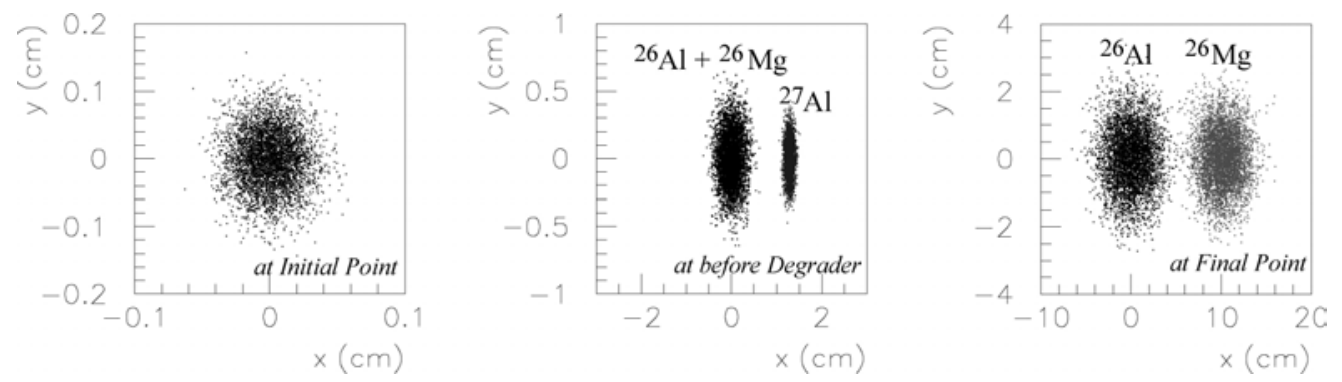

Figure 4 Result for separation of aluminum isotopes obtained by the TURTLE code. Explanations for these plots are given in the text.

Figure 4 shows the result of separation between aluminum and magnesium isotope using the TURTLE code. The figures are, from left to right, the beam profile at the initial point, before the degrader, and at the final point. The emittances of ${ }^{26} \mathrm{Al}$ after the energy degrader were $\varepsilon_{x}=220.40 \pi \mathrm{mm} \bullet \mathrm{mrad}$ and $\varepsilon_{y}=52.85 \pi \mathrm{mm} \bullet \mathrm{mrad}$ at the final point. The values of ${ }^{26} \mathrm{Mg}$ were $\varepsilon_{x}=184.97 \pi \mathrm{mm} \bullet \mathrm{mrad}$ and $\varepsilon_{y}=47.29 \pi \mathrm{mm} \bullet \mathrm{mrad}$ at the final point. The beam spot size of ${ }^{26} \mathrm{Al}$ and ${ }^{26} \mathrm{Mg}$ at the final point was $10.39 \times 5.38 \mathrm{~cm}$ and $9.35 \times 4.98 \mathrm{~cm}$ in the $\mathrm{x}-\mathrm{y}$ beam profile, respectively. As seen in the middle figure, ${ }^{27} \mathrm{Al}$ is clearly separated from a bundle of admixed ${ }^{26} \mathrm{Al}$ and ${ }^{26} \mathrm{Mg}$. The momentum difference between ${ }^{26} \mathrm{Al}$ and ${ }^{26} \mathrm{Mg}$ is only $0.012 \%$ for the magnetic rigidity and the mass difference is about $0.017 \%$. The energies of ${ }^{26} \mathrm{Al}$ and ${ }^{26} \mathrm{Mg}$ were $9.475 \mathrm{MeV}$ and $9.752 \mathrm{MeV}$, respectively, after the energy degrader with a $140 \mu \mathrm{g} / \mathrm{cm}^{2}(0.76 \mu \mathrm{m})$ beryllium foil. The momentum difference obtained was $1.44 \%$, corresponding to $11.3 \mathrm{~cm}$ in separation between these isotopes at the final position (see right figures in Figure 4). The separation between ${ }^{26} \mathrm{Al}$ and ${ }^{26} \mathrm{Mg}$ becomes clearer with increasing degrader thickness. At the same time, growth of the beam width is inevitable. 


\section{SUMMARY}

The new beam line at the SNU-AMS was designed by the TRANSPORT code for AMS counting the beryllium and aluminum nuclides. Separation of rare isotopes was simulated by the TURTLE code. For this simulation, AMS of aluminum as well as beryllium is feasible in the present design for the new beam line. In addition, the use of the energy degrader makes it possible to separate even a small mass difference in ${ }^{10} \mathrm{Be}-{ }^{10} \mathrm{~B}$ and ${ }^{26} \mathrm{Al}-{ }^{26} \mathrm{Mg}$ isotopes. Based on the present simulation study, construction of the new beam line is underway: The $100^{\circ}$ magnet was completely assembled and water cooling line is underway. We are also building another chamber for the $30^{\circ}$ dipole magnet.

\section{ACKNOWLEDGEMENTS}

The authors are deeply indebted to Dr John Southon for his enlightening comments. This work was supported by Korea Research Foundation Grant (KRF-2002-070-C00031).

\section{REFERENCES}

Brown KL, Rothacker F, Carey DC, Iseline Ch. 1973. PSI graphic transport framework by Rohrer U based on a CERN-SLAC-FERMILAB version. TRANSPORT, a computer program for designing charged particle beam transport system. Conseil Européen pour la Recherche Nucléaire 73-16.

Brown KL, Iseline Ch. 1974. PSI Graphic Turtle framework by Rohrer U based on a CERN-SLAC-FERMILAB version. DECAY TURTLE. Conseil Européen pour la Recherche Nucléaire 74-2.

Highland VL. 1975. Some practical remarks on multiple scattering. Nuclear Instruments and Methods in Physics Research B 129:497-9.

Kim JC, Youn M, Kim IC, Park JH, Song YM, Kang J, Choi HR. 2003. Present activities and future plan at Seoul National University AMS Facility. Journal of the Korean Physics Society 43:S45-9.

Kim JC, Park JH, Kim IC, Lee C, Cheoun MK, Kang J, Song YM, Jeong SC. 2001. Progress and protocol at the Seoul National University AMS Facility. Journal of the Korean Physical Society 39:778-82.

Kim JC, Lee CH, Kim IC, Park JH, Kang J, Cheoun MK, Kim YD, Moon CB. 2000. A new AMS facility in Korea. Nuclear Instruments and Methods in Physics Research B 172:13-7.

Livingood JJ. 1969. The Optics of Dipole Magnet, Chapter 2. New York: Academic Press

Tschalär C. 1968. Straggling distributions of extremely large energy loss. Nuclear Instruments and Methods B 64:237-43.

Tsai YS. 1974. Pair production and bremsstrahlung of charge leptons. Reviews of Modern Physics 46:81551.

Yun CC, Lee CS, Kim JC, Youn M. 2003. Beam line design and simulation for mass separation of beryllium isotopes at the AMS facility in Korea. 2003 IEEE International Conference on Plasma Science. Jeju, Korea, 2-5 June 2003. 